Methods Retrospective analysis of the Intensive Care National Audit and Research Centre database of all patients with cirrhosis admitted to ICU from January 2006 to November 2010. Statistical analysis was performed with SPSS V. 19. The area under receiver operating curve (AUROC) was used to assess the prognostic accuracy of the scoring models.

Results 135 out of 4890 (2.8\%) admissions had diagnosed cirrhosis. Mean age was $53.77 \%$ were white, $16 \%$ were Asian, and $7 \%$ were black. $74 \%$ were male. Cause of cirrhosis was alcohol in $70.4 \%$, HCV in $9.6 \%$, HBV in $4.4 \%$ and NASH in $5.2 \%$. Admission was for sepsis in $34 \%$ of patients, GI bleeding in $24 \%$, and encephalopathy in $12 \%$. Mortality was $39 \%$ in ICU, $55 \%$ at 30 days and $59 \%$ at 1 year. $30-$ day mortality in those who required renal replacement therapy (RRT), advanced cardiovascular support, mechanical ventilation or CPR was $75 \%, 65 \%, 58 \%$ and $70 \%$ respectively. Overall all-cause ICU mortality was $19.4 \%$ for this period.

Conclusion Mortality in this series compares favourably with published rates, particularly at 1 year. Mortality in patients who require CPR or RRT is high at $70 \%$ and $75 \%$, but is appreciably better than the $100 \%$ and $89 \%$ mortality previously reported. SOFA score has the best predictive value for mortality, but is not strongly predictive. All predictive scores have a worse AUROC for mortality than previously seen suggesting a reduced prognostic validity in the non-transplant UK setting.

Abstract PTU-039 Table 1 Predictors of 30-day mortality

\begin{tabular}{llll}
\hline Variable & $\begin{array}{l}\text { Area under } \\
\text { the curve }\end{array}$ & $\mathbf{9 5 \%} \mathbf{C l}$ & $\begin{array}{l}\text { Range of previously reported } \\
\text { area under the curve }\end{array}$ \\
\hline SOFA & 0.736 & 0.650 to 0.821 & $0.77-0.94$ \\
SAPS II & 0.716 & 0.629 to 0.802 & 0.80 \\
APACHE II & 0.697 & 0.606 to 0.788 & $0.77-0.90$ \\
MELD & 0.691 & 0.603 to 0.779 & $0.78-0.878$ \\
RIFLE score & 0.626 & 0.533 to 0.720 & 0.837 \\
\hline
\end{tabular}

Competing interests None declared.

\section{PTU-040 ALCOHOL: ALWAYS DETRIMENTAL TO THE IMMUNE SYSTEM? THE ROLE OF ACTIVE ALCOHOL CONSUMPTION ON NEUTROPHIL FUNCTION IN ALCOHOL-RELATED CIRRHOSIS}

doi:10.1136/gutjnl-2012-302514c.40

1J M Ryan, ${ }^{*}{ }^{1} \mathrm{~N}$ J Taylor, ${ }^{1} \mathrm{G}$ K Manakkat Vijay, ${ }^{1} \mathrm{~T}$ H Tranah, ${ }^{1} \mathrm{R} \mathrm{D}$ Abeles, ${ }^{2} \mathrm{~A}$ Riva, ${ }^{3} \mathrm{~N}$ Vergis, ${ }^{2} \mathrm{~L}$ Markwick, ${ }^{2} \mathrm{~S}$ Chokshi, ${ }^{1} \mathrm{~J}$ O'Grady, ${ }^{1} \mathrm{~J} \mathrm{~A}$ Wendon, ${ }^{1} \mathrm{Y} \mathrm{Ma}$, ${ }^{1} \mathrm{D}$ L Shawcross. ${ }^{1}$ Institute of Liver Studies and Transplantation, King's College London School of Medicine at King's College Hospital, London, UK; ${ }^{2}$ Foundation for Liver Research, London, UK; ${ }^{3}$ Hepatology Research Unit, St. Mary's Hospital, London, UK

Introduction Neutrophil dysfunction has been reported in patients with alcohol-related cirrhosis (ARC) and is associated with increased risk of infection and mortality. There is a paucity of understanding regarding the mechanisms of immune dysfunction in patients with active alcohol consumption and ARC. This study aimed to characterise neutrophil phenotype, functionality and plasma cytokine profiles in abstinent patients with ARC $(n=17)$ compared to those actively drinking $(\mathrm{n}=19)$, split by MELD score $<15$ vs $>15$ compared to healthy controls $(n=12)$.

Methods Neutrophils were isolated from patients with ARC. Phagocytic capacity was analysed by flow cytometry using FITClabelled Escherichia coli and oxidative burst (OB) was determined by the percentage of neutrophils producing reactive oxygen species (ROS) at rest, and after stimulation with opsonised $E$ Coli. Neutrophils were stained with anti-CD11b (APC-Cy7), -CD16 (PE) and -TLR4 (biotin-conjugated PE-Cy7 Streptavidin). Plasma cytokine profiling was performed using cytokine bead array.

Results Phagocytosis was significantly reduced in the cirrhotic groups compared to controls $(\mathrm{p}=0.02)$ however this was not influenced by MELD score or abstinence. Spontaneous OB was significantly increased in the cirrhotic groups compared to controls $(\mathrm{p}=0.03)$. Median spontaneous $\mathrm{OB}$ in the abstinent patients with MELD <15 was $26 \%$ [IOR 8-42] compared to $10 \%$ (IOR 7-44) in those actively drinking. Median spontaneous $\mathrm{OB}$ in the abstinent group with MELD>15 was 31\% (IOR 8-61) compared to $14 \%$ (IOR 4-29) in the active alcohol-drinkers. Stimulated burst was not impaired in the cirrhotic groups, with comparative values to controls. Plasma pro- and anti-inflammatory cytokine profiles were not discriminatory between the groups. Baseline TLR4 expression was increased in the MELD>15 abstinent group compared to active drinkers $(p=0.004)$; alcohol attenuated resting TLR4 expression to values seen in controls.

Conclusion Active alcohol consumption did not impact on neutrophil phagocytic capacity but reduced spontaneous OB by $50 \%$ with a reduction in the generation of ROS and decreased resting TLR4 expression. This supports a paradoxical anti-oxidant role of active alcohol drinking in patients with ARC that may promote endotoxin tolerance and warrants further investigation.

Competing interests None declared.

\section{PTU-041 ACUTE FATTY LIVER OF PREGNANCY: A REVIEW OF 20 CASES \\ doi:10.1136/gutjnl-2012-302514c.41}

K Axe, ${ }^{*}$ C Ch'ng. Gastroenterology, Singleton Hospital, Swansea, Swansea, UK

Introduction Acute Fatty Liver of Pregnancy (AFLP) is a life threatening condition occurring in the last trimester of pregnancy, its incidence is up to $1 / 1000$ births $^{1}$ and is commoner in primiparous women with male or twin pregnancies. ${ }^{2}$ It has features in common with HELLP syndrome, though coagulopathy, hypoglycaemia and polydipsia are commoner in AFLP and anaemia is not a feature. ${ }^{3}$ Liver biopsy confirms the diagnosis but a Swansea Score of 6 or above, in absence of another explanation, indicates AFLP. ${ }^{1}$ We aimed to review our experiences of AFLP to further our recognition and management of the condition.

Methods A retrospective review of 20 patients with suspected AFLP between 1993 and 2011, assessing patient demographics, Swansea score, biopsy results and complications.

Results Median age 26 (range 18-35), gestation age 36 (range $33-40$ ), 3 twin pregnancies and $65 \%$ male births. Most were G1P0. Average Swansea score 9 (range 6-13). Commonest presentation was abdominal pain, malaise and vomiting. $60 \%$ had polydypsia, $25 \%$ had encephalopathy. $100 \%$ had raised transaminase, AST $231 \mathrm{u} / 1$ (range 84-4019), ALT $274 \mathrm{u} / \mathrm{l}$ (range 99-722). 95\% had high urate $0.61 \mathrm{mmol} / 1$ (range $0.32-0.97$ ), $85 \%$ had high bilirubin $62 \mathrm{umol} / 1$ (range 13-192) and 85\% leucocytosis $19.4 \times 10^{9} / 1$ (range 12.8-74.5). 65\% had renal impairment, Creatinine $141 \mathrm{umol} / \mathrm{l}$ (range 40-305), 55\% had coagulopathy, PT 15 s (range 10-57), 40\% had hypoglycaemia, glucose $3 \mathrm{mmol} / 1$ (range $1-6$ ). $20 \%$ patients had high ammonia, average 58 (range 28-67), 65\% patients did not have ammonia checked. Ultrasound scan (USS) was performed on 4/ 20 patients, 2 had steatosis. $50 \%$ patients had biopsy, done 2-13 days post partum. All had microvesicular steatosis, 2 cholestasis, 1 centrilobular necrosis and 1 hepatocyte inflammation. All bloods, where available, showed improvement in 7 days and normalised by 3 months. Complications occurred in 3 pregnancies; 2 intrauterine deaths and 1 prolonged ITU stay. No patients had recurrence of AFLP. No maternal deaths occurred. 
Conclusion Male babies and twin pregnancies increased risk AFLP. A high index of suspicion is needed in patients with abdominal pain, malaise and high ALT in the last trimester, the Swansea score should be applied to aid diagnosis, higher Swansea scores indicate greater risk. Recent years have shown improved maternal and foetal survival $^{2}$ but correct identification and early obstetric intervention prevents complications such as liver failure.

Competing interests None declared.

\section{REFERENCES}

1. Ch'ng CL, Morgan M, Hainsworth I, et al. Prospective study of liver dysfunction in pregnancy in Southwest Wales. Gut 2002;51:876-80.

2. Knight M, Nelson-Piercy C, Kurinczuk JJ, et al. A prospective national study of acute fatty liver of pregnancy in the UK. Gut 2008;57:951-6.

3. Kingham JG. Liver disease in pregnancy. Clin Med 2006;6:34-40.

\section{PTU-042 DNA-PK OR ATM INHIBITION INHIBITS NON-HOMOLOGOUS END JOINING AND ENHANCES CHEMO- AND RADIO SENSITIVITY IN HEPATOCELLULAR CANCER CELL LINES}

doi:10.1136/gutjnl-2012-302514c.42

L Cornell, ${ }^{*} \mathrm{~J}$ Munck, N Curtin, H Reeves. Northern Institute for Cancer Research, Newcastle University, Newcastle upon Tyne, UK

Introduction Hepatocellular carcinoma (HCC) is chemotherapy resistance possibly due to dysregulation of DNA damage signalling and repair. DNA double-strand breaks (DSBs) are the most cytotoxic lesions induced by ionising radiation (IR) and anticancer drugs such as topoisomerase II poisons (eg, doxorubicin). DSBs are repaired by non-homologous end joining (NHEJ), initiated by DNAdependent protein kinase (DNA-PK), and homologous recombination (HR), reportedly initiated by Ataxia telangiectasia mutated (ATM). DNA-PK is up-regulated in HCC (GEO profiles), possibly contributing to anticancer therapy resistance. To assess DNA-PK and ATM as therapeutic targets for chemo- and radio-sensitisation in HCC we determined the effect of their inhibition in HCC cell lines.

Methods DNA-PK and ATM protein levels and activation by IR (Western blot), DSB levels (y-H2AX foci), HR (RAD51 foci), cell growth (DAPI fluorescence) and cytotoxicity (colony formation) following exposure to IR or doxorubicin was determined in a panel of 6 hepatoma cell lines (HepG2, Hep3B, Huh7, SNU-182, SNU475 and $\mathrm{PLC} / \mathrm{PRF} / 5)$. Studies were performed in the presence and absence of the DNA-PK inhibitor NU7441, and the ATM inhibitor KU55933.

Results DNA-PK protein concentration and activity were high in all HCC cell lines. In contrast, ATM expression varied, and was lowest in Hep3B cells. Cell-specific sensitivities to IR and doxorubicin correlated with ATM expression (highest in HepG2 and lowest in Hep3B). NU7441 sensitised all cells to doxorubicin (average $\mathrm{PF}_{50}$ $4.3 \pm 3.0$ ) and IR (average $\mathrm{PF}_{50} 3.9 \pm 1.1$ ), significantly increasing growth inhibition and reducing survival (4.8 to 3.3-fold; colony forming assays). KU55933 significantly potentiated cytotoxicity in HepG2 cells (eightfold) but had little effect on cytotoxicity in Hep3B cells. Following exposure to IR, both NU7441 and KU55933 delayed DSB repair ( $\sim 50 \%$ clearance yH2AX foci at $4 \mathrm{~h}$ vs only $10 \%-15 \%$ in presence NU7441). NU7441 also enhanced HR (threefold increase in RAD51 foci), while KU55933 had little effect.

Conclusion DNA-PK levels were high in all HCC cells and its inhibition with NU7441 was associated with significant chemo- and radio-sensitisation. Potentiation by ATM inhibition varied in the cell lines, reflecting the level of ATM expression. Both inhibitors substantially impaired the rapid phase of DNA repair commonly attributed to NHEJ. Notably, KU55933 had little effect on HR, suggesting that ATM is not central to this repair pathway. We propose that these inhibitors will increase the effectiveness of lower safer doses of cytotoxic therapies, amplifying tumour toxicity, and that DNA-PK and ATM levels in tumour and non-tumour liver will predict those patient likely to benefit.

Competing interests None declared.

\section{PTU-043 DEVELOPMENT OF A LIVER-SPECIFIC, TUMOUR- SELECTIVE RECOMBINANT ADENO-ASSOCIATED VIRUS VECTOR TO TARGET HEPATOCELLULAR CARCINOMA}

doi:10.1136/gutjnl-2012-302514c.43

${ }^{1} \mathrm{M}$ Della Peruta, ${ }^{*} \mathrm{C}$ Binny, ${ }^{2} \mathrm{D}$ Nathwani, ${ }^{2} \mathrm{~J}$ Mclntosh, ${ }^{2} \mathrm{C}$ Rosales, ${ }^{3} \mathrm{~A} \mathrm{M}$ Davidoff, ${ }^{1} \mathrm{R}$ Williams, ${ }^{1} \mathrm{~S}$ Chokshi, ${ }^{2} \mathrm{~A} \mathrm{C}$ Nathwani. ${ }^{1}$ The Institute of Hepatology, Foundation for Liver Research, London, UK; ${ }^{2}$ Department of Haematology, UCL Cancer Institute, London, UK; ${ }^{3}$ Department of Surgery, St. Jude Children's Research Hospital, Memphis, Tennessee, USA

Introduction Hepatocellular carcinoma (HCC) is the fifth most common cancer worldwide and is currently one of the most difficult to treat. Surgical resection and liver transplantation are considered curative therapies but they are feasible for only a small number of patients. Other therapeutic options, such as radiofrequency ablation and arterial chemoembolization, are effective only in small tumours. Recombinant adeno-associated virus (rAAV) vectors are ideally suited for gene transfer-based therapeutic approaches for HCC because of their safety profile and remarkable tropism for the liver, with $>90 \%$ of the vector particles being detectable in hepatocytes following a single systemic administration in non-human primates.

Methods Sequence complementarity to miR-122a (122aT) was cloned downstream of luciferase under the control of a new liver specific promoter/enhancer element, HLP, and flanked by the AAVinverted-terminal-repeats (ITRs). AAV8 capsid-pseudotyped AAV8HLP-LUC $\pm 122 \mathrm{aT}$ vector stocks were prepared by 293T-based threeplasmid transient transfection, and used for: in vitro transduction of HCC cell lines HUH-7 (miR-122a-positive) and SNU-387 (miR122a-negative); in vivo tail vein injection of tumour-bearing SCID and $\mathrm{B} 6$ mice.

Results A single tail-vein injection of rAAV8 in tumour-bearing mice resulted in selective transduction of the liver as well as of HCC, but not neuroblastoma xenografts. We cloned the 122aT into our expression cassette to improve the transgene expression for HCC, taking advantage of the differential expression of miR-122a (abundant in normal liver but down regulated in most HCC). Normalised transgene expression with AAV8-HLP-LUC-122aT was at least 26.5fold higher in SNU-387 when compared to HUH-7 cell lines. Tailvein injection of rAAV8-HLP-LUC resulted in high transgene expression in liver, but mice transduced with rAAV8-HLP-Luc-122aT had little or no luciferase expression despite the proviral DNA presence. Subcutaneous HCC xenografts in mice showed a strong luciferase signal following tail-vein injection of both of these vectors.

Conclusion Inclusion of $122 \mathrm{aT}$ in the expression cassette allows regulation of $\mathrm{rAAV}$ mediated transgene expression in HCC cell lines and normal liver, and reduces expression in miR-122a positive cells (eg, normal hepatocytes). This novel vector, therefore, has the potential to deliver therapeutic transgenes to HCC in preference to normal hepatocytes in the liver, thus limiting toxicity.

Competing interests None declared. 